\title{
Simulation optimization on sucking up dust particle by suction mouth in sanitation sweepers under the negative pressure
}

\author{
Jiaqiang $E^{1,2, a}{ }^{*}$, Qingguo Peng ${ }^{2, b}$, Teng Liü, c ${ }^{2, \text { Wei Zuo }}{ }^{2, d}$, Yi Huang ${ }^{1,2, e}$ \\ ${ }^{1}$ State Key Laboratory of Construction Machinery, Changsha 410013 , China \\ ${ }^{2}$ College of Mechanical and Vehicle Engineering, Hunan University, Changsha 410013 , China \\ aejiaqiang@126.com, bpengqingguo317@163.com, 'liuye0321@126.com,

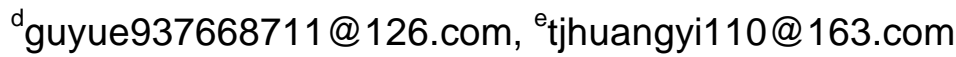

\begin{abstract}
Keywords: Sanitation sweepers; Negative pressure sucking up; Dust particle; Simulation optimization Abstract. A suction mouth model is established to research the negative pressure sucking up work process of medium dry brush type sanitation sweeper pneumatic conveying system. The flow field distribution and the dust particle motion residence trajectory are analyzed by numerical simulation, and then some optimization policies are proposed and verified. The result shows that the original suction mouth model have three problems which were solved by optimization policies. In addition, the sucking up velocity is increased by the optimization, but because of the special structure, the longest dust particle residence time is delayed 0.08 s compared with the original suction mouth model.
\end{abstract}

\section{Introduction}

Study showed that particle pollution was one of the main pollutants in city air pollution, while the industrial dust and traffic dust was the main source of particulate pollutants [1]. If these particulate pollutants cannot be treated properly, the city was likely to be "bad phenomenon fine ash, muddy on rainy days". Sanitation sweeper was the most important environmental sanitation machinery, it had strong adaptability, sweeping energy strong, long single operation time etc. [2].The suction mouth in sweeper pneumatic conveying system[3] was the most critical part, so determining its size and optimized could reduce the pressure loss of airflow, weaken the particle impact and improve the sweeper cleaning efficiency. Since twentieth Century 40's, some foreign enterprises in developed countries began to study and produce sweeper[4]. Researches on China's sweeping car were done since twentieth Century 60 years[5-7].Now most of the sweeper adopts the latter, but if the back blowing type suction mouth design improperly, it can not achieve the ideal effect of absorbing dust, and may lead to strong fugitive dust [8].Today although road sweeper have been studied for decades at home and aboard, but these studies mainly is through the enterprise using engineering practice means to complete, according to the fluid mechanics analysis, this paper mainly research and analyze the sweeper suction mouth's flow field pickup role of entrainment process for particulate contamination by negative pressure suction, which can provide a foundation for the research and optimization of sweeper pneumatic conveying system.

\section{Model}

\section{Physical model}

This paper mainly studies the unilateral anti-blowing suction mouth, the mouth is divided into two layers, firstly, the gas blows to partition through the mouthpiece, then blows out from one end of the partition not sealed by changing direction. A unilateral anti-blowing suction mouth is generally applied to medium dry sweeper to absorb the smaller size garbage, the model structure is shown in Figure 1. 


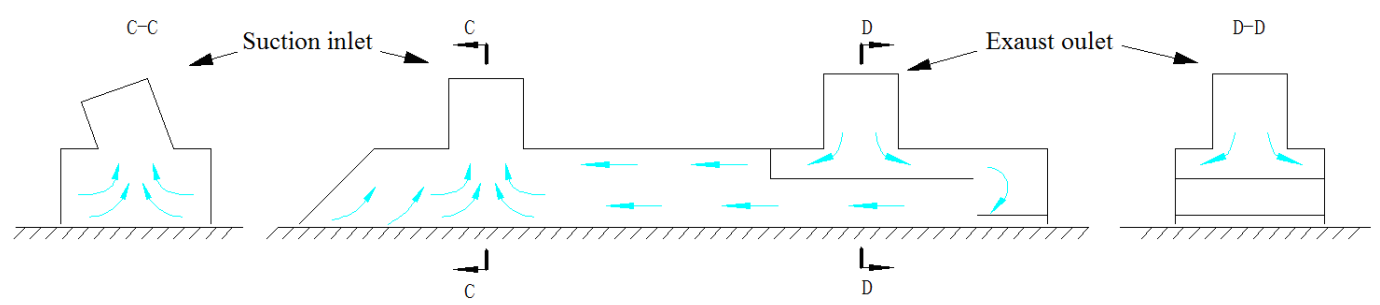

Figure 1 Two-dimensional graph of suction mouth

In the Figure1, the middle is the main view of suction mouth, left for the left view A-A cross section, right for the right view B-B section. Entire mouth has the length of $2000 \mathrm{~mm}$, width of $300 \mathrm{~mm}$, height of $180 \mathrm{~mm}$, is $10 \mathrm{~mm}$ away from the ground, and can absorb dust particles the maximum diameter is $10 \mathrm{~mm}$. In the process of this kind of suction mouth work, mouth will inhale dust to vacuum pipeline, and the vast majority of dust will be sent into the dustbin through the centrifugal dust collector, a small amount of dust is again back to the flow field of suction mouth through anti mouthpiece, then is inhaled by suction inlet through the pipeline in the Figure 1 model, thus avoiding the second fugitive dust, achieving the higher purpose of purify.

\section{Mathematical model}

Suction mouth needs to follow the theorem of conservation of nature in the work process, and the dust starting in the negative pressure sucking needs to follow grain motion equation. The negative pressure sucking the granular bed flow of suction nozzle is turbulent flow in this paper, and the analysis does not involve energy release and absorption, so only considering the mass conservation, momentum conservation, the turbulent kinetic energy equation and the dust particles starting equation.

\section{Optimization and validation}

As the simulation analysis on the previous model, data cloud of Figure 2 Figure 6 can get by simulating similarly to the model above. Figure 2 shows the distribution of internal flow field of the improved nozzle, the partial enlargement of the two ends in Figure 3 and Figure 4.

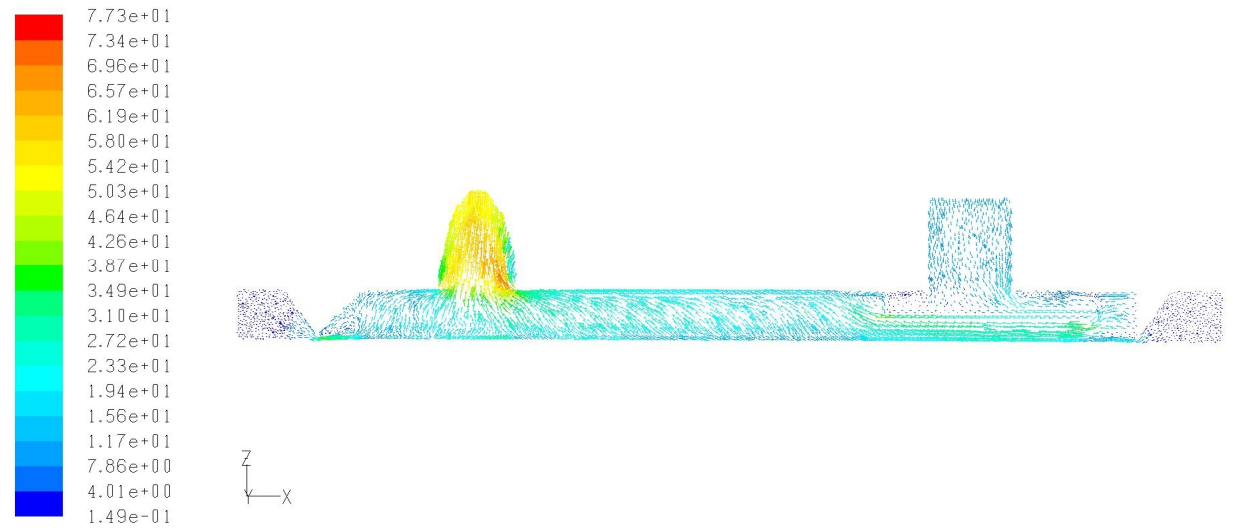

Figure 2 Internal flow field distribution of $\mathrm{XZ}$ plane

As shown in Figure 2, it can be found that the speed of air sucked into the suction nozzle increased through suction nozzle structure transformation, which can maintain sucking efficiency based on decrease the negative pressure, thereby saving energy; and the suction nozzle can pick up dust particles from the right side (near the side of the exhaust port), this point was also reflected in Figure 4, but the speed of picking up is low, about $20 \mathrm{~m} / \mathrm{s}$, with which it is difficult to pick up the iron particles of larger diameter, but it still able to pick up common dust on most roads. As can be seen from Figure 3, there was still a vortex area at the left of suction nozzle, but the eddy zone is smaller than before without improvement, the gas velocity at this eddy zone was lower than the end, which had no impact of concussion on dust of inhalation. 

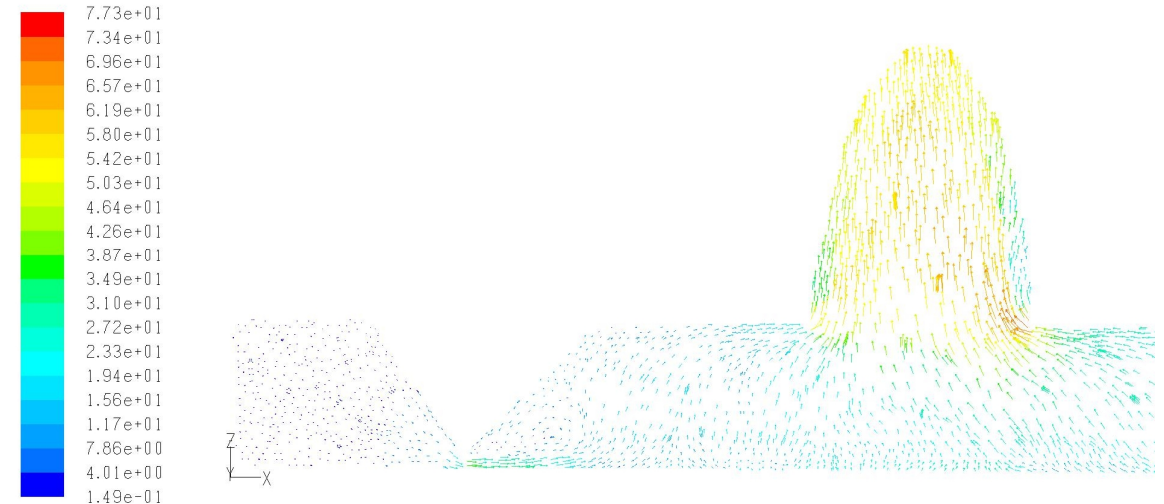

Figure 3 New partial enlargement of vacuum end flow field distribution

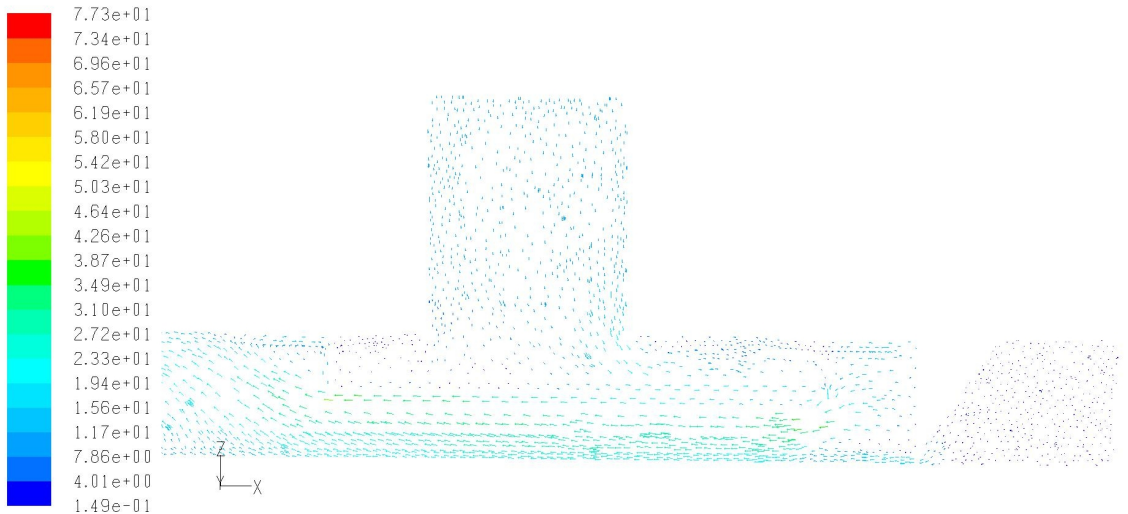

Figure 4 New partial enlargement of exhaust end flow field distribution

Figure 5 shows the distribution of flow field of suction nozzle improved in section C-C. The nozzle tilted 20 degrees to rear (Y direction), from the graph it can be observed that the flow field of the front of nozzle was significantly higher than the rear, so the new nozzle can suck dust better in the process of work .

Figure 6 reflected the movement and residence time of internal dust in the improved suction mouth in the process of working. From the Figure it can be found that the longest retention time of internal dust in suction nozzle was $1.47 \mathrm{~s}, 0.08 \mathrm{~s}$ higher than before without improvement, the main reasons for this is because the exhaust port add a partition, the speed of dust particle sucked into from the right of suction nozzle is very slow, and path was longer, so the residence time in the ceiling part of the mouth was longer.
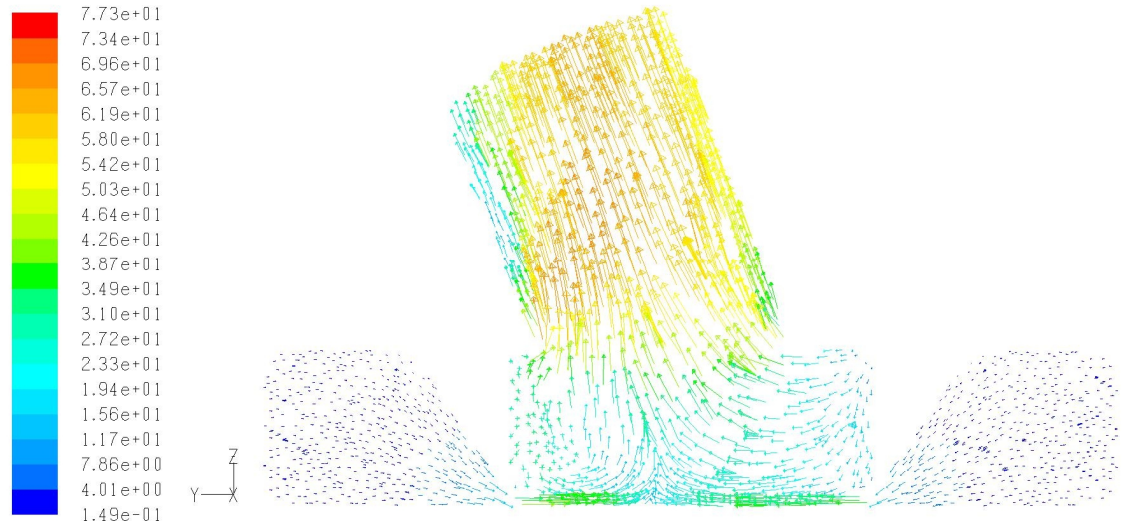

Figure 5 Flow field distribution of $\mathrm{C}-\mathrm{C}$ plane 


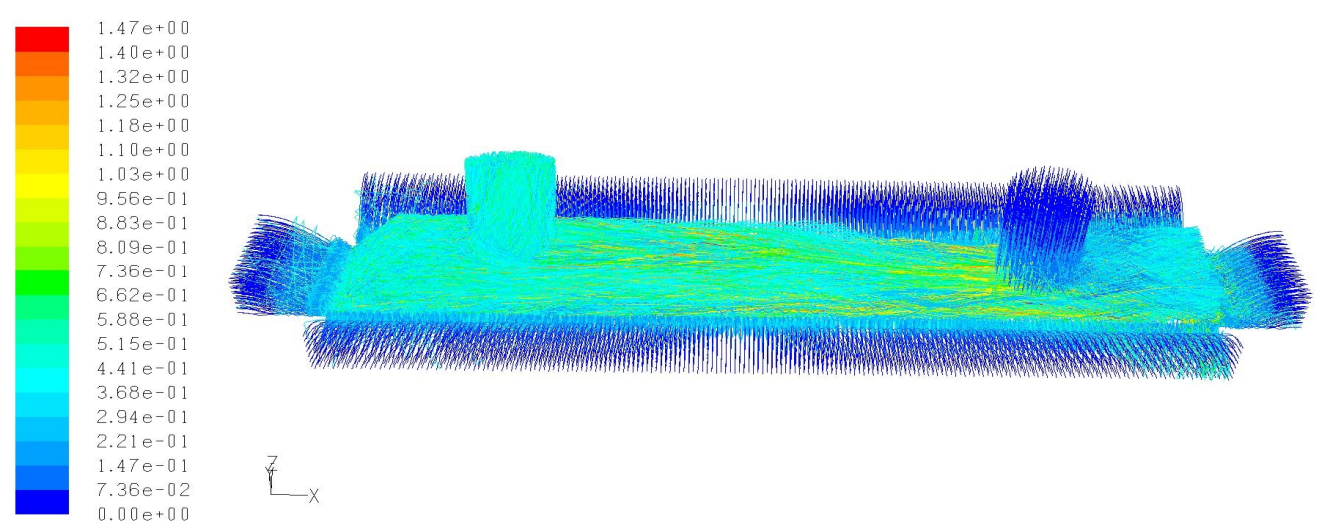

Figure 6 Trajectory and residence time of dust particle in the new suction mouth

\section{Conclusion}

(1) The sucking zone at the front of old suction nozzle was smaller than the rear, and sweeping vehicle mainly absorbed dust front of itself in the process of advance. The inlet was inclined $20^{\circ}$ toward the rear of mouth from the vertical, the results obtained show that it can enlarge the sucking region front of mouth, thus improving the absorbing efficiency.

(2) The speed of entrainment of improved in most region of mouth had improved, which can maintain the original sucking efficiency under the condition of decrease the negative pressure, thereby saving energy.

(3) The longest time of residence of dust particle in original nozzle was1.39s, while the longest time of residence of dust particle in improved nozzle was $1.47 \mathrm{~s}$. The reason for this phenomenon was mainly adding a baffle at the end of the right of suction nozzle.

\section{Acknowledgements}

The authors would like to acknowledge Project (SKLCM2014-8) supported by State Key Laboratory of Construction Machinery.

\section{References}

[1]Sun Yong. Design optimization and simulation analysis on pneumatic system on vacuum sweeper[D]. Northeastern University, 2008. (In Chinese)

[2]Jiang Zhaowen, Chen Kai, Geng Yuming. Nozzle system performance analysis for suction broom road sweeper[J]. Special Purpose Vehicle, 6 (2012) 92-97.

[3]Jiaqiang E, Teng Liu,Wei Zuo, Yi Huang. Simulation on entrainment process of suction mouth under negative[J]. Applied Mechanics and Materials, 711 (2015) 86-90.

[4]Ning Wenxiang. The five development trends of sweeper in foreign countries[J]. Special Purpose Vehicle, 4 (2010) 31-32.

[5]Luo Shanmao. Sweep machine research and development in China[J]. Construction Machinery Technology \& Management. 5 (2005) 46-48. (In Chinese)

[6]R A Jajko, O M Julien. Dirt deflector for cleaning heads[P].United States: 4807327

[7]D P Strauser, R E Field. Street sweeper pick-up head[P].United States: 5839157

[8]Jiaqiang E, Wei Zuo, Qingguo Peng, Shuhui Wang, Hao Zhu, Teng Liu, Yuanwang Deng, Jinke Gong. Variable flow area suction nozzle for road sweeping vehicle[P].China: ZL201510104259.5, 2015 